\title{
Chronic Pain - Can It Be Cured?
}

\section{Adams JD}

\author{
USC School of Pharmacy, Los Angeles, USA
}

${ }^{*}$ Corresponding author: Adams JD, USC School of Pharmacy, Los Angeles, CA 90089-9121 USA, Tel: 323-442-1362,E-mail: jadams@usc.edu

Citation: Adams JD (2017) Chronic Pain - Can It Be Cured? J Pharm Drug Devel 4(1): 105. doi: 10.15744/23489782.4.105

Received Date: November 10, 2017 Accepted Date: November 20, 2017 Published Date: November 21, 2017

\begin{abstract}
Chronic pain is currently treated with several drugs and therapies that can be helpful, but usually do not cure chronic pain. In fact, many drug therapies make the pain worse or are toxic to the patient. Chronic pain treatment frequently involves oral or injected drugs because the perception is that chronic pain comes from an internal source. Chronic pain, and all other forms of pain, should be treated in the skin. Mechanisms of induction of chronic pain will be discussed and skin targets for the treatment and cure of chronic pain will be presented.

Keywords: Chronic pain; Opioids; NSAIDs; Plant medicines

List of Abbrivations: COX2: cyclooxygenase-2; NSAIDs: nonsteroidal anti-inflammatory drugs; IL-17 interleukin-17: TRP: transient receptor potential cation channel; $\gamma \delta$ T cells: skin resident T cells; TNF $\alpha$ : tumor necrosis factor $\alpha$; AMPA $\alpha$ : amino-3-hydroxy-5-methyl4-isoxazolepropionic acid
\end{abstract}

\section{Introduction}

Chronic pain can be treated with radiofrequency ablation therapy. In this procedure, lidocaine is injected at sites near the lumbar spine to find nerves that are responsible for pain transmission. The responsible nerves are then ablated with radiofrequency waves. This procedure relieves chronic back pain until the nerves recover, usually within 15 months. Chronic pain can be treated with pain shots administered to internal nerves that are involved in conducting pain signals. There are many possible injection sites such as trigger points in muscles, nerve roots along the spinal cord and spinal cord epidural sites. These shots contain local anesthetics or steroids. Pain shots can relieve pain for as much as one year. Opioids are the drugs of choice for chronic pain, especially hydrocodone, methadone and oxycodone. Opioids are usually used orally to deliver the drugs to internal sites. Opioids can also be used by injection or dermal patches. Non-steroidal anti-inflammatory drugs (NSAIDs) are also used, but are usually considered to not be potent enough to adequately treat chronic pain. NSAIDs are used orally to deliver the drugs to internal sites.

Complementary therapies are also available for chronic pain including acupuncture. It is well known that acupuncture relieves pain for several hours. Yoga, massage and spinal adjustment are also used in chronic pain. Many chronic pain patients complain that yoga and massage can make their pain worse.

None of the drugs or procedures currently used actually cure chronic pain. Perhaps better internal sites for drug or radiofrequency wave administration can be found that will actually cure chronic pain. Perhaps better drug delivery systems will be able to provide better therapy that will cure chronic pain.

\section{The Opioid Crisis}

The healthcare community is either so confident in current therapy or knows of nothing better, that opioids continue to be prescribed for chronic pain. Opioids are addictive, cause tolerance and kill patients due to respiratory depression and seizures. In 2017, the Centers for Disease Control report that 15,000 patients died from oxycodone, methadone and hydrocodone use. Patients who are tolerant to these drugs search for stronger alternatives including heroin and fentanyl. Heroin is about twice the potency of morphine. Fentanyl is about 100 times more potent than morphine. In 2017, 15,000 patients died from heroin injection. Heroin use has become a middle class epidemic. In 2017, 20,000 patients died from fentanyl injection. Fentanyl use is also a middle class epidemic. This adds up to about 50,000 patients dying yearly from opioid use for pain.

When tolerance to opioids occurs, the dose is increased. As the dose escalates, opioids begin to cause pain, opioid induced hyperalgesia. This increase in pain does not occur in every patient. The mechanism of opioid induced hyperalgesia is under 
investigation, but may involve the induction of chemokines in the skin [1]. Clearly, in order to cure chronic pain, opioid administration must be stopped.

\section{The NSAIDs Crisis}

Much less reported in the literature is the NSAIDs crisis. The FDA increased black box warnings for all NSAIDs in July 2015. The black box warnings indicate that NSAIDs cause heart attacks, strokes and other thrombus problems. The FDA indicates that the risk of heart attack or stroke is increased by at least $10 \%$ by NSAIDs. It can be assumed that all people in the US use NSAIDs sometime during any year. Therefore, it can be calculated that NSAIDs are responsible for at least 24,000 deaths from heart attack and 14,000 deaths from stroke. The Centers for Disease Control report that NSAIDs cause 100,000 ulcers yearly, of which 16,500 die. This adds up to 54,500 deaths every year from NSAIDs. The US public might be alarmed to learn that the NSAID pain relievers sold over the counter are so dangerous.

\section{Mechanisms Involved in Chronic Pain}

Damage to the skin results in the release of chemokines (Figure 1) by damaged neurons, dendritic cells and other cells [2,3]. Neuronal chemokines bind to their specific G protein coupled receptors on macrophages, which activates macrophages and induces cyclooxygenase-2 (COX2, 4). Inflammatory factors are then produced by macrophages. Macrophages secrete chemokines that bind to their specific receptors on sensory neurons and increase the transactivation of transient receptor potential cation (TRP) channels, which enhances pain [5]. TRP channels are the most abundant pain receptors in the body and are mostly located on skin sensory neurons [6]. Prostaglandins, including PGI2 and PGE2, secreted by macrophages and other cells, interact with prostaglandin receptors, such as EP2 and IP, on sensory neurons causing pain, and increase the activation of TRP channels enhancing pain. Prostaglandins also up regulate the production of chemokines by dendritic cells [7]. Opioid peptides secreted in the skin by leukocytes and other cells induce the production of chemokines and their receptors [1]. $\gamma \delta \mathrm{T}$ cells are skin resident T cells that secrete IL-17, which induces chemokine production. IL-23 and IL-1 $\beta$ secreted by macrophages stimulate IL-17 production [8,9]. Tumor necrosis factor $\alpha(\mathrm{TNF} \alpha)$ released by damaged keratinocytes stimulates IL-17 production [10]. Chemokines released by damaged dendritic cells activate $\gamma \delta$ T cells [11]. All skin sensory neurons use glutamate as their primary neurotransmitter. Calcium permeable $\alpha$-amino-3-hydroxy-5-methyl-4-isoxazolepropionic acid (AMPA) receptors on peripheral sensory neurons regulate chronic pain [12]. Other extracellular molecules involved in chronic pain in the skin include: bradykinin, histamine, nerve growth factor, 4-hydroxynonenal, ATP, 5,6-epoxyeicosatrienoic acid, 5-hydroxyeicosa-tetraenoic acid, calcitonin gene-related peptide, high mobility group protein B1. All of these factors are released following damage, pain and inflammation. This can establish chronic pain in the skin. Chronic pain involves a cyclic, self-perpetuating mechanism in which chemokines stimulate production of prostaglandins and IL-17. Prostaglandins and IL-17 then stimulate chemokine production.

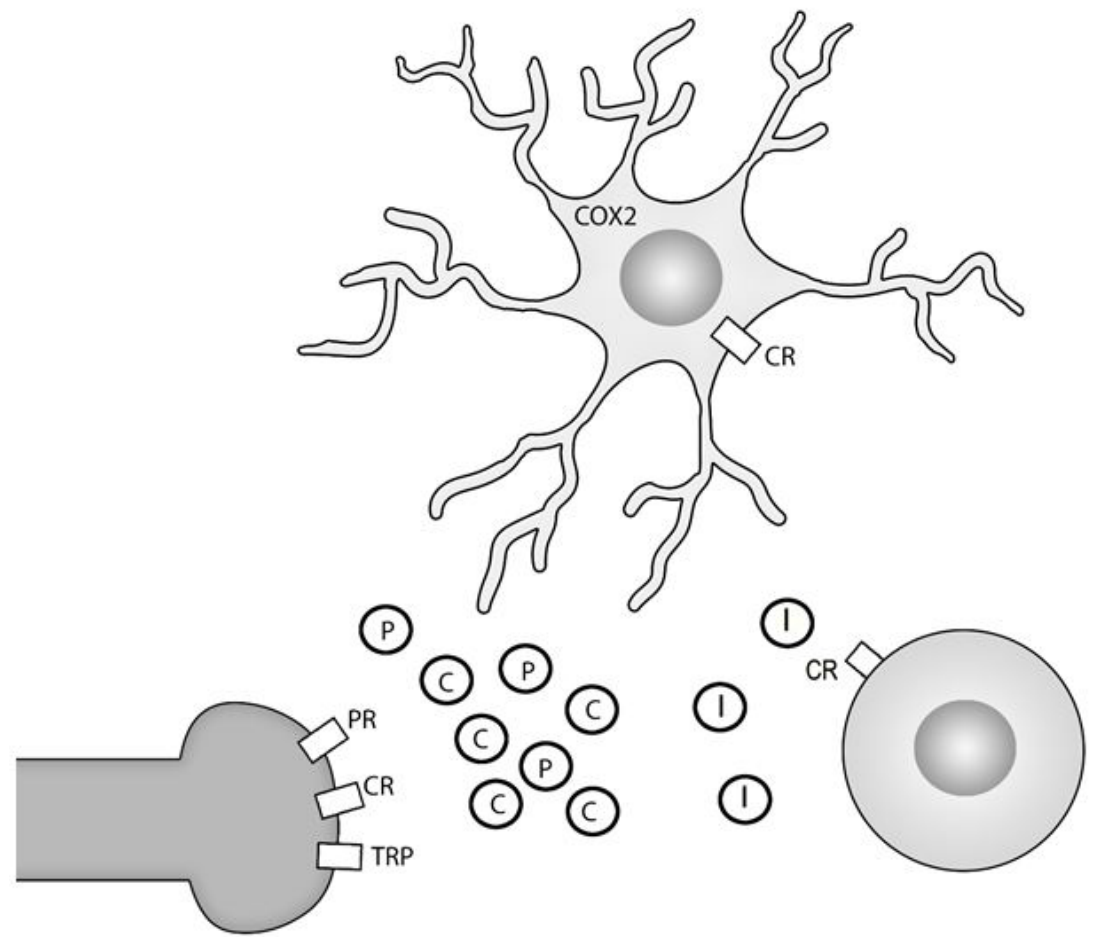

Figure 1: Chronic pain mechanisms - A damaged sensory nerve terminal in the skin releases chemokines (C) that attract macrophages, bind to $\mathrm{C}$ receptors (CR) and induce COX2 in macrophages. Macrophages secrete $\mathrm{C}$ that transactivate neuronal TRP channels. Prostaglandins $(\mathrm{P})$ are released by macrophages and bind to their receptors (PR) to cause pain. Resident $\mathrm{T}$ cells in the skin bind C and secrete interleukin-17 (I) that induces more $\mathrm{C}$ production. Figure modified from [15] 
Journal of Pharmaceutics \& Drug Development

Of course, the brain and brain stem are involved in chronic pain mechanisms also. The involvement of the brain and brain stem in chronic pain has been adequately discussed in several publications [1,5]. Despite many years of attempting to cure chronic pain with systemically administered drugs that penetrate into the brain and brain stem, there is no cure. In fact, the most notable effects of the systemic approach to chronic pain treatment has been the deaths of over 100,000 patients yearly and the hospitalization of thousands more.

\section{Potential Targets for Curing Chronic Pain}

Clearly, there are several potential targets for drug therapy in chronic pain in the skin. The skin is where pain is sensed. The safest and most effective treatment for pain is in the skin $[13,14]$. Chronic pain is probably caused by the mechanisms discussed above in the skin. Treating the skin may be the best approach to curing chronic pain.

COX2 is induced in the skin during chronic pain. COX2 inhibition in the skin can be easily accomplished with methyl salicylate that can be applied to the skin and penetrates into the skin. Temporary inhibition of skin COX2 by methyl salicylate does not cure chronic pain. It may be better to down regulate COX2 in the skin in order to cure chronic pain [15]. Several natural products are known to penetrate into the skin after topical application and down regulate COX2 in the skin, including sesquiterpenes [15] and flavonoids such as eupafolin [16].

TRP channels are targets that may lead to cures for chronic pain. There are several products on the market that are applied to the skin and relieve pain by inhibiting TRP channels. These products contain monoterpenoids, 10 carbon compounds from plants, which penetrate into the skin. However, inhibition of TRP channels by these products is inadequate to cure chronic pain, perhaps because too few monoterpenoids are used. Distinct TRP channels exist in non-overlapping populations of skin sensory neurons. There are at least 28 different TRP channels, each with different sensitivities to monoterpenoids [6]. A mixture of monoterpenoids may provide better pain therapy through inhibition of many different TRP channels. Rather than using a silver bullet to cure chronic pain, a cocktail of monoterpenoids should be used. Monoterpenoids can inhibit IL-17 production in the skin [17]. When IL-17 is no longer produced, chemokine synthesis may stop. This may cure chronic pain.

Curing chronic pain might be possible through down regulation of chemokine or opioid peptide production in the skin. IL-23 and IL-1 $\beta$ down regulation might cure chronic pain. Calcium permeable AMPA receptors may be targets for chronic pain cure.

The endocannabinoid, anandamide, activates cannabinoid-1 receptors on keratinocytes. This binding inhibits the mechanistic target of rapamycin, which is a protein kinase. The end-result is that neighboring resident T cells decrease their secretion of IL-17 [18]. Various plant medicines made from Cannabis sativa contain cannabinoids and are currently on the market for application to the skin. Perhaps one of these products cures chronic pain.

\section{References}

1. Melik Parsadaniantz S, Rivat C, Rostene W, Reaux-Le Goazigo A (2015) Opioid and chemokine receptor crosstalk: a promising target for pain therapy? Nature Rev Neurosci 16: 69-78.

2. de Haas A, van Weering H, de Jong E, Boddeke H, Biber K (2007) Neuronal cytokines: versatile messengers in central nervous system cell interaction. Mol Neurobiol 36: 137-51.

3. Abboud D, Hanson J (2016) Chemokine neutralization as an innovative therapeutic strategy for atopic dermatitis. Drug Discov Today 22: 702-11.

4. Jones R, Kelly R, Critchley H (1997) Chemokine and cyclooxygenase-2 expression in human endometrium coincides with leukocyte accumulation. Hum Reprod 12: $1300-6$.

5. Ji R, Xu Z, Gao Y (2014) Emerging targets in neuroinflammation-driven chronic pain. Nature Rev Drug Disc 13: 533-48.

6. Moran M, McAlexander M, Biro T, Szallasi A (2011) Transient receptor potential channels as therapeutic targets. Nature Rev Drug Disc 10: 601-20.

7. McIlroy A, Caron G, Blanchard S, Fremaux I, Duluc D, Delneste Y, Chevalier A, Jeannin P (2006) Histamine and prostaglandin E2 up regulate the production of TH2 attracting chemokines (CCL17 and CCL22) and down regulate IFN-gamma induced CXCL10 production by immature human dendritic cells. Immunol 117: $507-16$

8. Heath W, Carbonne F (2013) The skin-resident and migratory immune system in steady state and memory: innate lymphocytes, dendritic cells and T cells. Nature Immunol 14: 978-85.

9. Verreck F, de Boer T, Langenberg D, Hoeve M, Kramer M, et al. (2004) Human IL-23-producing type 1 macrophages promote but IL-10-producing type 2 macrophages subvert immunity to (myco)bacteria. Proc Natl Acad Sci USA 101: 4560-5.

10. Stein J, Nombela-Arrieta C (2005) Chemokine control of lymphocyte trafficking: a general overview. Immunol 116: 1-12.

11. Thaiss C, Semmling V, Franken L, Wagner H, Kurts C (2011) Chemokines: a new dendritic cell signal for T cell activation. Frontiers Immunol 2: 1-9.

12. Gangadharan V, Wang R, Ulzhöfer B, Luo C, Bardoni R, et al. (2011) Peripheral calcium-permeable AMPA receptors regulate chronic inflammatory pain in mice. J Clin Invest 121: 1608-23.

13. Adams J (2016) The effects of yin, yang and qi in the skin on pain. Medicines 3:5

14. Adams J, Wang X (2015) Control of pain with topical plant medicines. Asian Pacific J Trop Biomed 5: 93-5.

15. Adams J, Haworth I, Coricello A, Perri F, Nguyen C, et al. (2017) The treatment of pain with topical sesquiterpenes. Front Nat Product Chem, Bentham Science Publishers, Sharjah, 176-95.

16. Lee C, Lin Z, Hsu L, Fang J, Chiang Y, et al. (2016) Eupafolin ameliorates COX2 expression and PGE2 production in particulate pollutants exposed human keratinocytes through ROS/MAPKs pathways. J Ethnopharmacol 189: 300-9. 
17. Games E, Guerreiro M, Santana F, Pinheiro M, de Oliveira E, et al. (2016) Structurally related monoterpenes p-cymene, carvacrol and thymol isolated from essential oil from leaves of Lippia sidoides Cham. (Verbenaceae) protect mice against elastase-induced emphysema. Molecules 21: 1390-1407.

18. Chiurchiu V, Rapino C, Talamonti E, Leuti A, Lanuti M, et al. (2016) Anandamide suppresses proinflammatory T cell responses in vitro through type 1 cannabinoid receptor mediated mTOR inhibition in human keratinocytes. J Immunol 197: 3545-53.

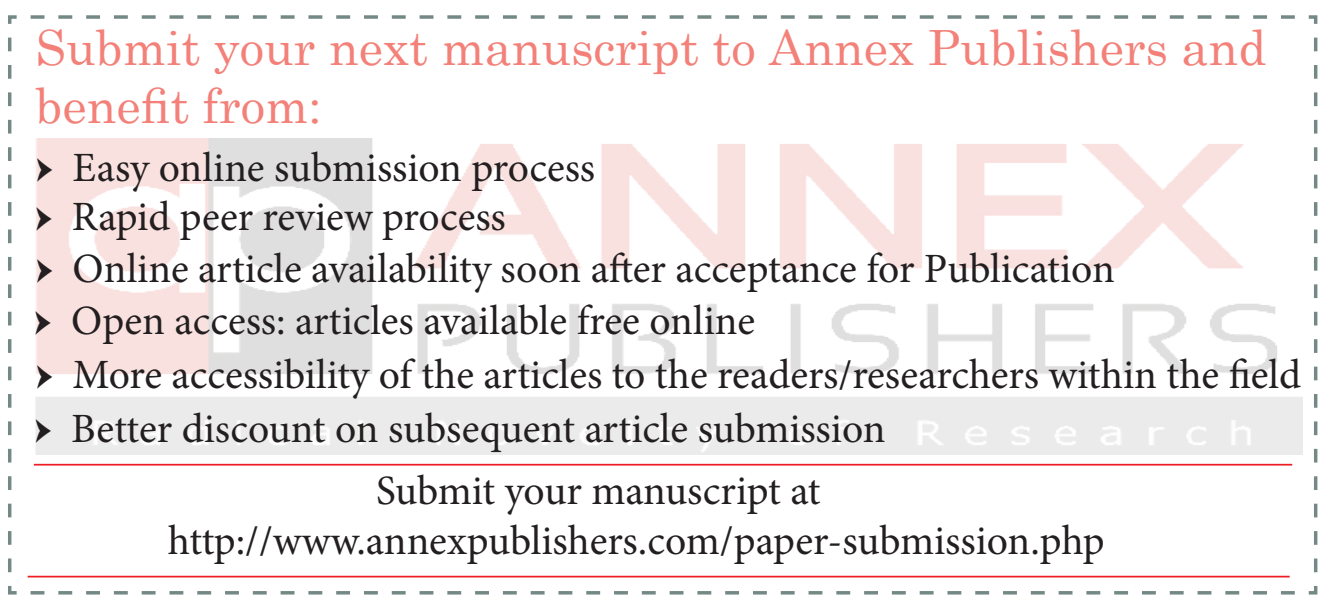

